Vol. 33, Issue 6, December 2006

Editorial

473 Perinatal Transfusion Medicine - Immunological Aspects

Barz, D. (Jena)

Review Articles

474 Reproductive Immunology - an Update

Poehlmann, T.G.; Fitzgerald, J.S. (Jena); Busch, S.; Schleussner, E. (Jena);

Gutiérrez, G. (Jena/Buenos Aires); Blois, S.; Arck, P.C. (Berlin);

Kämmerer, U. (Würzburg); Szekeres-Bartho, J. (Pecs);

Markert, U.R. (Jena)

486 Impact of the Antiphospholipid Syndrome on Complications during Pregnancy

Schleussner, E. (Jena)

495 Controversy: HLA-Antibodies Responsible for Fetal/Neonatal Alloimmune Thrombocytopenia an Update

Panzer, S. (Wien)

Original Articles

501 Immunotherapy with Paternal Lymphocytes for Recurrent Miscarriages and Unsuccessful in vitro Fertilization Treatment

Wegener, S.; Schnurstein, K.; Hansch, S.; Bolz, M.; Briese, V. (Rostock); Sudik, R. (Neubrandenburg); Wegener, R.; Busecke, A.;

Müller, H. (Rostock)

509 Efficacy and Safety of the New Intravenous Immunoglobulin IGIV 10\% in Adults with Chronic Idiopathic Thrombocytopenic Purpura

Varga, G. (Szeged); Volková, Z. (Prague); Leibl, H. (Vienna); Gasztonyi, Z. (Györ); Hlusi, A. (Olomouc); Mayer, J. (Brno); Chojnowski, K. (Lodz); Wolf, H.H. (Halle); Sharkhawy, M.; Pavlova, B.G.; Birthistle, K.; Engl, W.; Walter, S.; Ehrlich, H.J. (Vienna); ITP-IGIV 10\% Study Group

515 Neonatal RBC Transfusions - Comparison of Two Patterns

Podraza, W.; Nowak, J.; Domek, H.; Czajka, R.; Rudnicki, J.; Kordek, A.; Gonet, B. (Szczecin)
Band 33, Heft 6, Dezember 2006

Editorial

473 Perinatale Transfusionsmedizin - immunologische Aspekte Barz, D. (Jena)

Übersichtsarbeiten

474 Reproduktionsimmunologie - ein Überblick Poehlmann, T.G.; Fitzgerald, J.S. (Jena); Busch, S.; Schleussner, E. (Jena); Gutiérrez, G. (Jena/Buenos Aires); Blois, S.; Arck, P.C. (Berlin); Kämmerer, U. (Würzburg); Szekeres-Bartho, J. (Pecs); Markert, U.R. (Jena)

486 Der Einfluss des Antiphospholipidsystems auf Komplikationen während der Schwangerschaft Schleussner, E. (Jena)

495 Gibt es die fötale/neonatale Alloimmunthrombozytopenie infolge einer Immunisierung gegen HLAKlasse-I-Antigene? - eine Aktualisierung Panzer, S. (Wien)

Originalarbeiten

501 Immuntherapie mit paternalen Lymphozyten bei wiederholten Aborten und erfolgloser In-vitroFertilisationsbehandlung

Wegener, S.; Schnurstein, K.; Hansch, S.; Bolz, M.; Briese, V. (Rostock); Sudik, R. (Neubrandenburg); Wegener, R.; Busecke, A.;

Müller, H. (Rostock)

509 Die Wirksamkeit und Sicherheit von IGIV 10\%, einem neuen Immunglobulin zur intravenösen Anwendung, bei Erwachsenen mit chronischer idiopathischer thrombozytopenischer Purpura

Varga, G. (Szeged); Volková, Z. (Prague); Leibl, H. (Vienna); Gasztonyi, Z. (Györ); Hlusi, A. (Olomouc); Mayer, J. (Brno); Chojnowski, K. (Lodz); Wolf, H.H. (Halle); Sharkhawy, M.; Pavlova, B.G.; Birthistle, K.; Engl, W.; Walter, S.; Ehrlich, H.J. (Vienna); ITP-IGIV 10\% Study Group

515 Neonatale RBC-Transfusionen - zwei Methoden im Vergleich

Podraza, W.; Nowak, J.; Domek, H.; Czajka, R.; Rudnicki, J.; Kordek, A.; Gonet, B. (Szczecin)

\begin{tabular}{ll}
\hline KARGER & ( 2006 S. Karger GmbH, Freiburg \\
Fax +4976145207 14 & Accessible online at: \\
$\begin{array}{l}\text { E-mail Information@Karger.de } \\
\text { www.karger.com }\end{array}$ & www.karger.com/tmh
\end{tabular}


Vol. 33, Issue 6, December 2006

520 A Prospective Crossover Trial Comparing Performance and in vitro Platelet Quality of Three New Apheresis Devices with Current Equipment Picker, S.M.; Radojska, S.M.; Gathof, B.S. (Köln)

Society Bulletins

528 Recommendations for Thrombocyte Transfusion of the Thrombocyte Working Group of the DGTI, GTH and DGHO

Greinacher, A. (Greifswald); Kiefel, V. (Rostock);

Klüter, H. (Mannheim); Kroll, H. (Dessau); Pötzsch, B. (Bonn); Riess, H. (Berlin)

544 Innovations

546 News / Ticker

547 Meetings and Conferences

500 Imprint

550 Acknowledgement to Reviewers

551 Author Index Vol. 33, 2006

553 Subject Index Vol. 33, 2006

III Contents Vol. 33, 2006 (following page 556)

Forthcoming Papers are listed on page 549.

\section{Band 33, Heft 6, Dezember 2006}

520 Performance und In-vitro-Plättchenqualität dreier neuer Apheresegeräte im prospektiven Crossover-Vergleich zu existierendem Equipment

Picker, S.M.; Radojska, S.M.; Gathof, B.S. (Köln)

Society Bulletins

528 Empfehlungen zur Thrombozytentransfusion der Thrombozyten-Arbeitsgruppe der DGTI, GTH und DGHO

Greinacher, A. (Greifswald); Kiefel, V. (Rostock);

Klüter, H. (Mannheim); Kroll, H. (Dessau); Pötzsch, B. (Bonn); Riess, H. (Berlin)

544 Innovationen

546 News / Ticker

547 Tagungen und Kongresse

500 Impressum

550 Dank an die Gutachter

551 Autorenverzeichnis Band 33, 2006

555 Sachwortverzeichnis Band 33, 2006

VI Inhalt Band 33, 2006 (nach Seite 556)

Einen Ausblick auf den Inhalt der kommenden Hefte finden Sie auf Seite 549. 